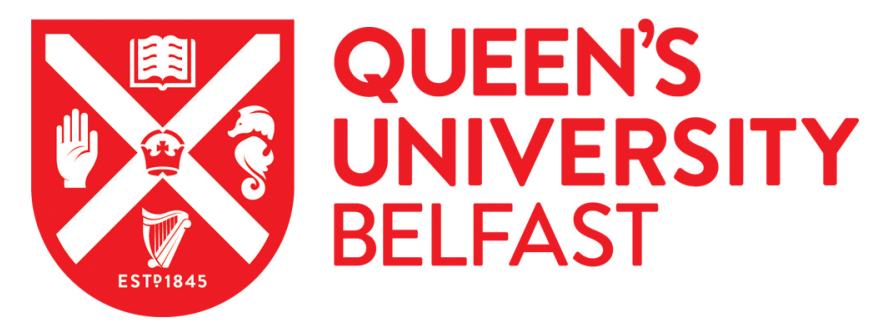

\title{
Systematic optimization of poly(vinyl chloride) surface modification with an aromatic thiol
}

McCoy, C., Irwin, N., Hardy, JG., Kennedy, S., Donnelly, L., Cowley, J., Andrews, G., \& Pentlavalli, S. (2017). Systematic optimization of poly(vinyl chloride) surface modification with an aromatic thiol. European Polymer Journal, 97, 40-48. https://doi.org/10.1016/j.eurpolymj.2017.09.030

\section{Published in:}

European Polymer Journal

\section{Document Version:}

Peer reviewed version

\section{Queen's University Belfast - Research Portal:}

Link to publication record in Queen's University Belfast Research Portal

\section{Publisher rights}

Copyright 2017 Elsevier.

This manuscript is distributed under a Creative Commons Attribution-NonCommercial-NoDerivs License

(https://creativecommons.org/licenses/by-nc-nd/4.0/), which permits distribution and reproduction for non-commercial purposes, provided the author and source are cited.

\section{General rights}

Copyright for the publications made accessible via the Queen's University Belfast Research Portal is retained by the author(s) and / or other copyright owners and it is a condition of accessing these publications that users recognise and abide by the legal requirements associated with these rights.

Take down policy

The Research Portal is Queen's institutional repository that provides access to Queen's research output. Every effort has been made to ensure that content in the Research Portal does not infringe any person's rights, or applicable UK laws. If you discover content in the Research Portal that you believe breaches copyright or violates any law, please contact openaccess@qub.ac.uk. 


\title{
Systematic optimization of poly(vinyl chloride) surface modification with an aromatic thiol
}

Colin P. McCoy*, Nicola J. Irwin, John G. Hardy ${ }^{\text {a }}$, Susan J. Kennedy, Louise Donnelly, John F. Cowley, Gavin P. Andrews, Sreekanth Pentlavalli

School of Pharmacy, Queen's University Belfast, Belfast BT9 7BL, UK

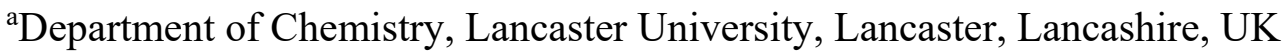

*Corresponding author

Tel: +44 (0) 2890972081; Fax: +44 (0) 2890247794; Email:c.mccoy@qub.ac.uk

\begin{abstract}
The efficient covalent functionalization of poly(vinyl chloride) (PVC), which is widely used in medical device manufacture, allows an array of potential property-enhancing surface modifications to be made. To demonstrate a general method of functionalization via substituted (functional) thiols, we describe a systematic approach to the optimization of PVC surface modification by nucleophilic substitution with 4-aminothiophenol through control of reaction conditions: solvent composition, sonication, reaction time and presence of base and/or phase transfer catalyst (PTC). Efficient thiol attachment was confirmed using solidstate NMR and Raman spectroscopies, and the extent of surface modification was quantified using ATR-FTIR spectroscopy. Sonicated samples exhibited a lower degree of modification than their statically immersed counterparts (21.7 vs $\left.99.6 \mu \mathrm{g} \mathrm{cm}^{-3}\right)$, and mechanical integrity was compromised. In DMSO/ $\mathrm{H}_{2} \mathrm{O}$ systems with a PTC, resultant degrees of PVC surface modification were up to $12.5 \%$ higher when caesium carbonate was employed as the base than in corresponding systems with potassium carbonate.
\end{abstract}

\section{Keywords}

PVC, thiol, nucleophilic substitution, surface modification 


\section{Introduction}

Poly(vinyl chloride) (PVC) is one of the most extensively employed thermoplastic materials worldwide, with more than 31 million tons produced per annum [1]. The diverse applications of PVC, for example within transportation, construction, packaging and healthcare, result from its chemical and biological stability, relatively low cost and ready processability [2]. PVC currently accounts for almost $30 \%$ of all disposable polymeric devices used in medical screening, diagnosis, treatment and care [3].

Modification of the surface of PVC has been investigated as an approach to reduce leaching of additives into the surrounding media [4], aid polymer recycling [5], improve the mechanical and interfacial properties for applications with biofluid contact [6,7], and prevent biofouling, a phenomenon of major importance for medical grade PVC [8]. A variety of physical and chemical processes have been explored for PVC surface modification, including oxygen and argon plasma treatment [9], graft polymerization of polymer brush layers [7], UV irradiation [10], and nucleophilic substitution by wet-chemical treatment [11]. Aromatic thiols have been successfully employed as nucleophilic agents for the substitution of chlorine atoms on the PVC backbone in solution, aqueous suspensions or in the melt [12-14]. Sacristan et al. (2000) gave the first report of the importance of solvent conditions, in particular the solvent/non-solvent ratio, on the resultant degree of surface selectivity in PVC modification reactions with sodium azide and aminothiophenol [13]. To-date, however, the importance of an extended range of tuneable reaction conditions on the extent of PVC surface modification has not been systematically investigated. In particular, the ability to dramatically alter the surface chemistry of PVC through simple, efficient immersion chemistry, would afford opportunities to develop a range of novel surface properties for, among other applications, drug release, prevention of bacterial attachment or improving biocompatibility. This study, employing 4-aminothiophenol as a model thiol, provides the first methodical investigation of the effects of solvent composition, sonication, presence of a base and phase transfer catalyst (PTC), and reaction times on the degree of modification at the PVC surface during wet-chemical treatment of polymer films, and ultimately presents a platform for optimised PVC functionalization.

\section{Experimental}

\subsection{Materials}


All reagents were used as received. 4-Aminothiophenol was purchased from Apollo Scientific Limited, UK. Caesium carbonate and tetrabutylammonium bromide (TBAB) were obtained from Sigma Aldrich, UK. Potassium carbonate, $N, N$-dimethylformamide (DMF), dimethyl sulfoxide (DMSO), ethylene glycol and diethyl ether were purchased from VWR International, UK. Unplasticised PVC films with a thickness of $0.2 \mathrm{~mm}$ were obtained from Goodfellow Cambridge Limited, UK.

\subsection{Methods}

Reaction conditions were varied as described below and the resulting polymers characterised by spectroscopic methods to determine the corresponding degrees of surface modification.

\subsubsection{Wet-Chemical Treatment of PVC Films}

\subsubsection{Effect of Solvent/Non-Solvent Ratio}

Unplasticised PVC film surfaces were modified by an adaptation of the methodology of Sacristan et al. (2000) [13]. Briefly, PVC samples $(3 \times 3 \mathrm{~cm})$ were added individually to solutions of DMF/ $\mathrm{H}_{2} \mathrm{O} 4: 1,5: 1$ and $6: 1(50 \mathrm{~mL})$ with 4-aminothiophenol $(530.5 \mu \mathrm{L}, 5$ $\mathrm{mmoL})$, and potassium carbonate $(0.692 \mathrm{~g}, 5 \mathrm{mmoL})$ or caesium carbonate $(1.629 \mathrm{~g}, 5$ $\mathrm{mmoL}$ ) as the base, and maintained at $60^{\circ} \mathrm{C}$ for $6 \mathrm{~h}$. Samples were then washed thoroughly with deionised water. Residual nucleophile and solvent were removed by washing for $24 \mathrm{~h}$ with diethyl ether $(3 \times 100 \mathrm{~mL})$, and samples were dried in vacuo prior to characterisation.

\subsubsection{Effect of Sonochemistry}

PVC samples were immersed in $\mathrm{DMF} / \mathrm{H}_{2} \mathrm{O}$ solutions $(50 \mathrm{~mL})$ containing potassium carbonate as the base and 4-aminothiophenol, as before, and sonicated at ambient temperature for $6 \mathrm{~h}$ (VWR Ultrasonic Cleaner, $230 \mathrm{~V}, 100 \mathrm{~W}$ ). Samples were washed and dried as previously described.

\subsubsection{Effect of Phase Transfer Catalyst}


PVC samples were immersed in $\mathrm{DMF} / \mathrm{H}_{2} \mathrm{O}$ solutions $(50 \mathrm{~mL})$ containing caesium carbonate or potassium carbonate as the base and 4-aminothiophenol modifying agent, as previously described. After addition of the PTC, TBAB (59.7 mg, $0.19 \mathrm{mmoL})$, solutions were maintained at $60^{\circ} \mathrm{C}$ for $6 \mathrm{~h}$. Deionised water was used to quench the reaction prior to washing and drying the modified PVC samples as before.

\subsubsection{Effect of Solvent Selection}

PVC surface modification reactions were carried out as before but using solvent systems containing THF, ethylene glycol or DMSO in place of DMF.

\subsubsection{Characterisation}

\subsubsection{Fourier Transform Infrared (FTIR) Spectroscopy}

Modified film surfaces were characterised by attenuated total reflectance (ATR) Fourier transform infrared (FTIR) spectroscopy. Spectra are an average of 12 scans acquired using an FT/IR-4100 spectrophotometer (Jasco, Great Dunmow, UK) equipped with a Pike MIRacle ATR accessory with diamond crystal at a resolution of $4 \mathrm{~cm}^{-1}$. Infrared spectra of the 4-aminothiophenol modifying agent and unmodified (control) PVC films were obtained between $\mathrm{NaCl}$ windows and in the ATR mode, respectively. The degree of surface modification was determined from a calibration curve prepared using standard solutions of 4aminothiophenol in methanol. The line equation determined from the calibration curve was y $=0.0002 \mathrm{x}-0.0057\left(\mathrm{r}^{2}=1.00\right)$. Absorbance values of modified films at $1602 \mathrm{~cm}^{-1}$, characteristic of aromatic ring stretching vibrations, were converted into surface concentrations $\left(\mathrm{mg} \mathrm{cm}^{-3}\right)$ by rearranging the line equation to make $\mathrm{x}$ the subject and multiplying by the depth of penetration into PVC $(1.33 \mu \mathrm{m})$.

\subsubsection{Raman Spectroscopy}

Raman spectra of modified films were obtained using a RamanStation R3 (Avalon Instruments, Belfast, UK) coupled with a RamanMicro 300 Raman microscope (PerkinElmer, Waltham, MA, USA) at 50x magnification. Raman scattered light from a $785 \mathrm{~nm}$ laser was 
collected between $3200-400 \mathrm{~cm}^{-1}$ at a resolution of $2 \mathrm{~cm}^{-1}$ and total exposure time of $20 \mathrm{~s}$.

\subsubsection{Solid-State NMR Analysis}

Solid-state ${ }^{13} \mathrm{C}$ spectra of modified and unmodified (control) PVC samples $(2 \times 2 \mathrm{~mm})$ were obtained using a Varian VNMRS spectrometer operating at $100.56 \mathrm{MHz}$.

\subsubsection{Tensile Analysis}

Tensile analysis was performed using a Stable Micro Systems TA-XT Plus Texture Analyser (Goldaming, Surrey, UK) in conjunction with Texture Exponent 32 software. Rectangular samples $(55 \times 15 \mathrm{~mm})$ of modified and unmodified (control) PVC films, with a thickness of $200 \mu \mathrm{m}$, were fixed between mobile upper and static lower clamps. The thickness of samples was not significantly altered by the surface modification process. The upper clamp was vertically elevated at a constant crosshead velocity of $1.0 \mathrm{~mm} \mathrm{~s}^{-1}$ until fracture. Ultimate tensile strength, Young's modulus and percentage elongation at break were determined from resultant plots of stress versus strain.

\subsubsection{Statistical Analysis}

Measurements were obtained at least in triplicate and results are expressed as mean $\pm \mathrm{SD}$. Statistical analysis was performed using GraphPad InStat software. Comparisons of individual mean values were made using the Kruskal-Wallis test, with post-hoc comparisons evaluated using Dunn's post-test. $P<0.05$ was used to denote significance.

\section{Results and Discussion}

PVC was herein surface-functionalized with reactive thiol moieties via nucleophilic substitution of chlorine atoms of the PVC backbone with 4-aminothiophenol, as outlined in Scheme 1, under various reaction conditions of solvent composition, time, sonication, base and PTC. 


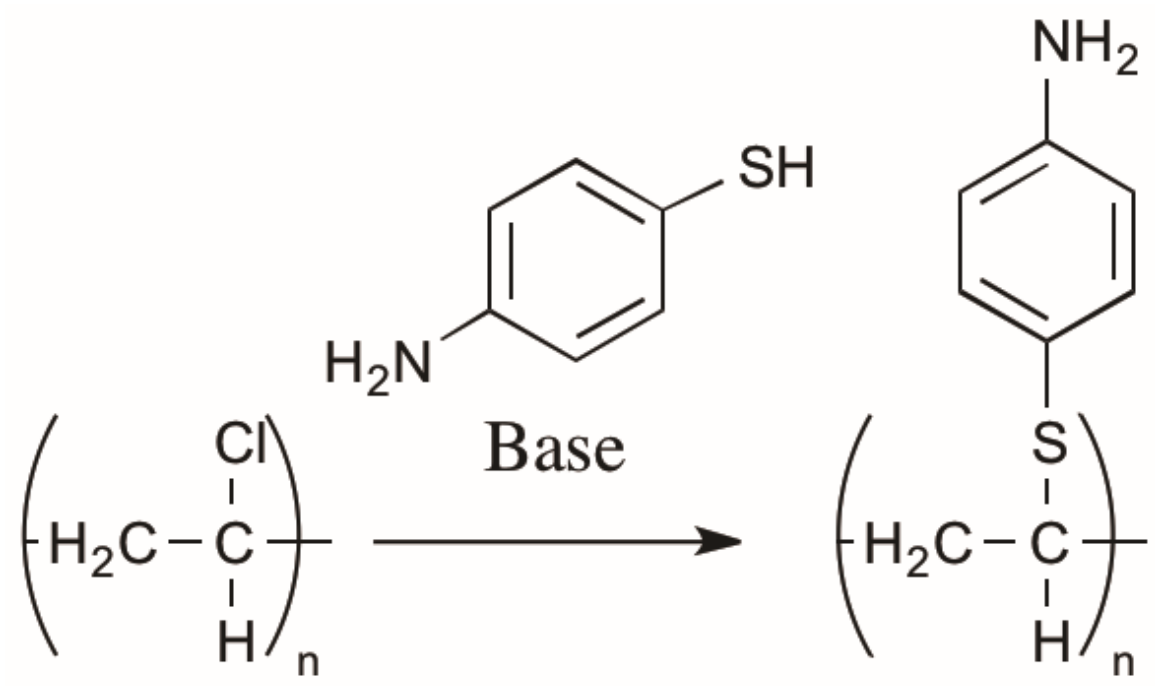

Scheme 1: Nucleophilic substitution of C-Cl group of PVC with 4-aminothiophenol.

\subsection{Spectroscopic Characterisation of Modified PVC Films}

Surfaces of PVC films covalently modified with 4-aminothiophenol in $\mathrm{DMF} / \mathrm{H}_{2} \mathrm{O}$ 5:1, with potassium carbonate as the base, were characterised by ATR-FTIR, Raman and NMR spectroscopies.

\subsubsection{FTIR Spectroscopy}

Overlaid ATR-FTIR spectra of the 4-aminothiophenol modifying agent, unmodified (control) PVC and modified PVC are presented in Fig. 1. The spectrum of 4-aminothiophenolmodified PVC reveals the presence of additional bands not observed in the spectrum of control PVC. These bands, which are also present in the spectrum of the 4-aminothiophenol reactant, result from the presence of the covalently-bound aromatic modifier at the material surface, and can be ascribed to aromatic primary amine $\mathrm{N}-\mathrm{H}$ stretching $\left(3470\right.$ and $\left.3350 \mathrm{~cm}^{-1}\right)$, aromatic C-C stretching (1600 and $1500 \mathrm{~cm}^{-1}$ ), aromatic C-H stretching ( 3100 and $3028 \mathrm{~cm}^{-1}$ ) and para-substituted C-H out-of-plane bending $\left(825 \mathrm{~cm}^{-1}\right)$ modes of vibration of the attached aromatic thiol moiety $[12,15]$. Additional bands in the spectrum of modified PVC at 1620 and $1180 \mathrm{~cm}^{-1}$ are ascribed to $\mathrm{N}-\mathrm{H}$ scissoring and C-N stretching vibrations, respectively, of the amino moiety of 4-aminothiophenol [15]. 


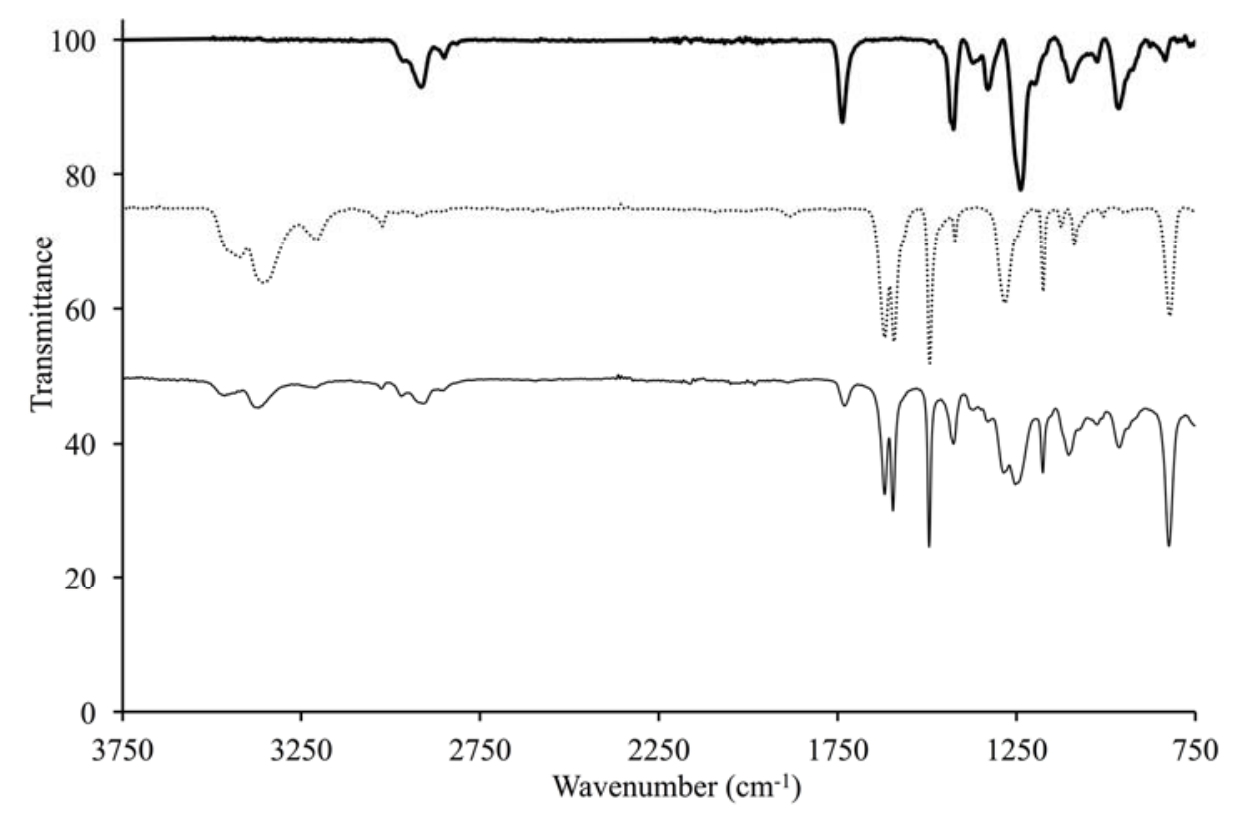

Fig. 1. ATR-FTIR spectra (3750-750 $\left.\mathrm{cm}^{-1}\right)$ of 4-aminothiophenol (...), unmodified (control) PVC (-), and 4-aminothiophenol-modified PVC (-). Transmittance values are offset for clarity.

\subsubsection{Raman Spectroscopy}

Overlaid Raman spectra of control and modified PVC are displayed in Fig. 2. The Raman spectrum of 4-aminothiophenol-modified PVC shows distinct changes in comparison to control PVC, confirming successful tethering of 4-aminothiophenol to the PVC surface. Two new bands assigned to aromatic C-H stretching vibrations (3054 and $2973 \mathrm{~cm}^{-1}$ ), an additional band ascribed to amino $\mathrm{C}-\mathrm{N}$ stretching vibrations $\left(1620 \mathrm{~cm}^{-1}\right)$, two bands corresponding to aromatic C-C stretching vibrations (1595 and $1496 \mathrm{~cm}^{-1}$ ), and an intense band at $1095 \mathrm{~cm}^{-1}$ with shouldering of the band at $616 \mathrm{~cm}^{-1}$ corresponding to C-S stretching vibrations in the spectrum of 4-aminothiophenol-modified PVC indicate the presence of the covalently-bound aromatic thiol modifying agent at the polymer surface. 


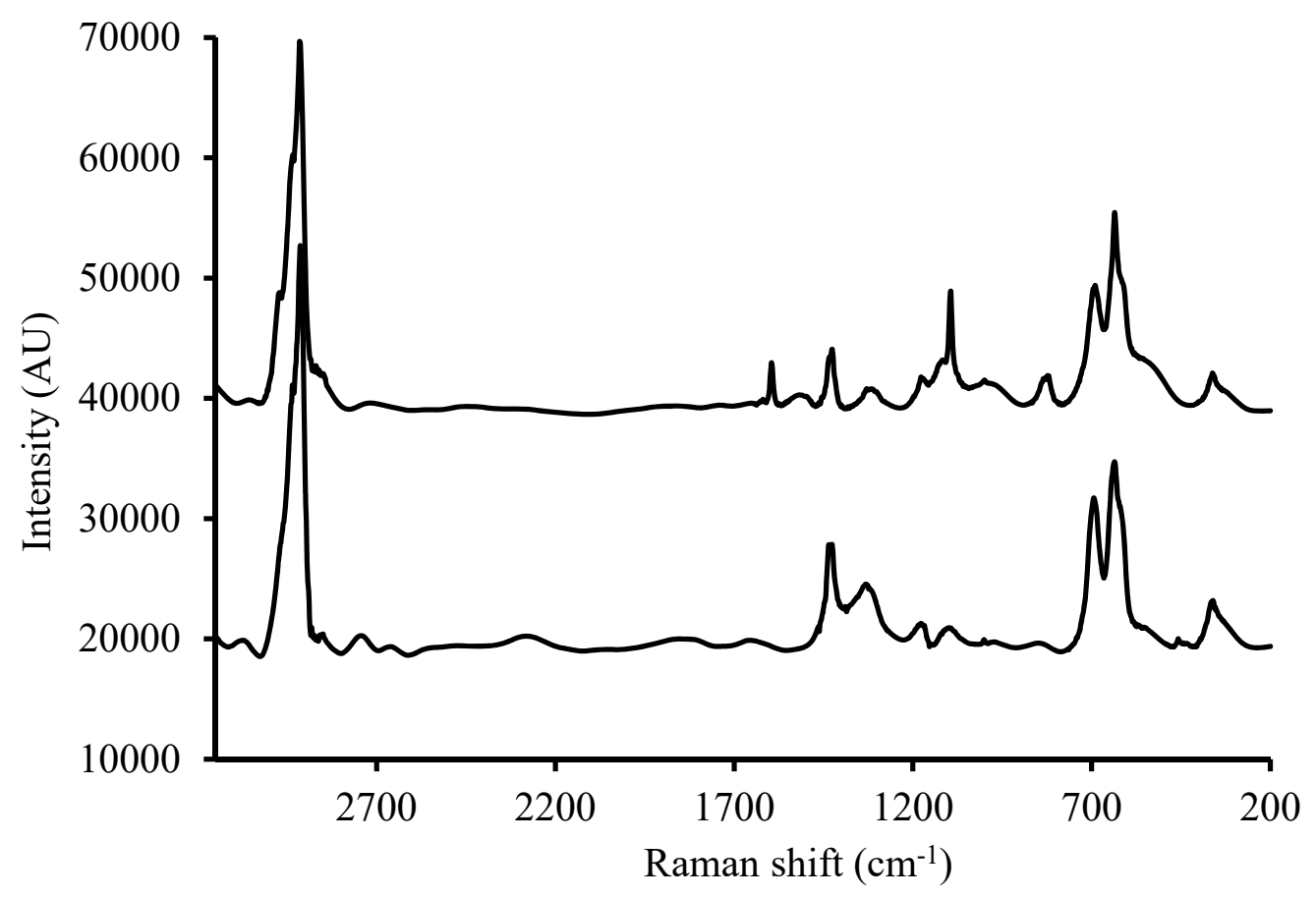

Fig. 2. Raman spectra of 4-aminothiophenol-modified PVC (top line) and unmodified (control) PVC (bottom line).

\subsubsection{NMR Spectroscopy}

The ${ }^{13} \mathrm{C}$ spectrum of 4-aminothiophenol-modified PVC exhibited analogous signals to that of control PVC, with some variation in intensity. Additional signals at 116, 138 and $148 \mathrm{ppm}$ in the ${ }^{13} \mathrm{C}$ spectrum of modified PVC were consistent with signals observed at 115, 134 and 147 ppm during NMR analysis of 4-aminothiophenol in solution. A difference spectrum demonstrated a reduction in the intensity of the signal at $57 \mathrm{ppm}$, indicative of the shift for $\mathrm{CH}-\mathrm{Cl}$, and a concomitant increase in intensity of the signals at 16 and $22 \mathrm{ppm}$, ascribed to terminal methyl and adjacent methylene groups, respectively, upon modification, thus confirming successful substitution of chlorine atoms with aromatic thiol moieties.

\subsection{Effect of Varying Reaction Conditions}

In order to demonstrate an optimised method for the functionalization of PVC with thiol moieties, an array of reaction conditions, including sonication, base, presence of a PTC, solvent composition and reaction times, were herein varied and the effects of each reaction variable on the subsequent degree of thiol attachment are reported below. 


\subsubsection{Effect of Sonication}

Interactions between the 4-aminothiophenol modifying agent and the PVC polymer chains lead to physical crosslinking of the polymer network, and are reported to limit the extent of PVC surface modification during wet-chemical treatment. This is due to the restricted mobility, and subsequent access, of the 4-aminothiophenol moiety to the chlorine atoms [12]. As such, the use of sonication as a method for attaining mass transfer of the reaction components at conditions of ambient temperature was investigated with concomitant changes in solvent compositions. Fig. 3 shows the resultant degrees of surface modification $\left(\mathrm{mg} \mathrm{cm}^{-3}\right)$ following static immersion (at $60^{\circ} \mathrm{C}$ ) and sonication (at ambient temperature) of PVC samples in $\mathrm{DMF} / \mathrm{H}_{2} \mathrm{O} 4: 1,5: 1$ and 6:1 systems (containing 20\%, 16.7\% and 14.3\% water respectively), with potassium carbonate as the base. In order to assess homogeneity of the modified PVC surface, five points were analysed and the mean values are displayed in Fig. 3, with error bars representing surface heterogeneity.

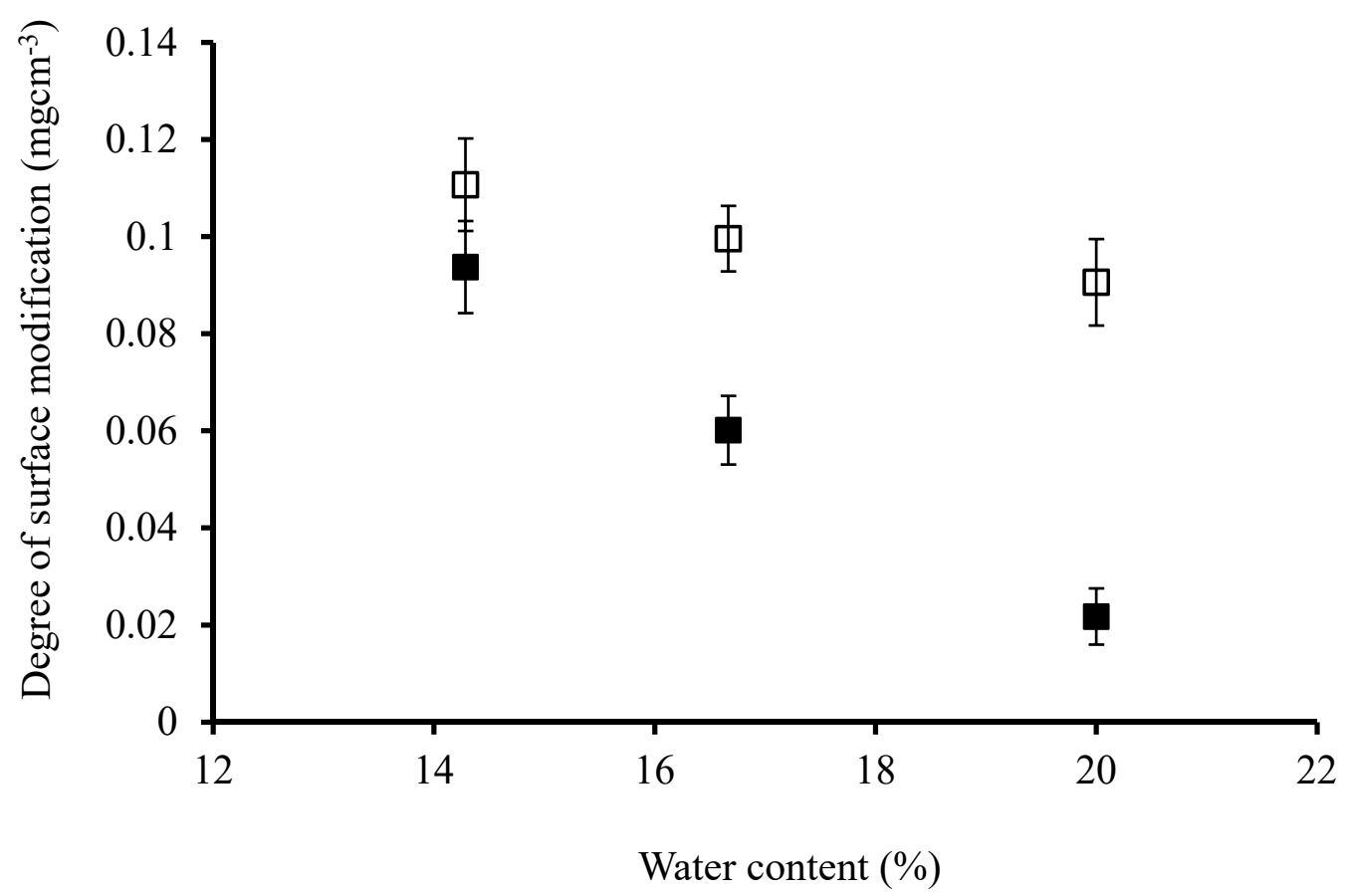

Fig. 3. Effect of sonication (at ambient temperature) (a) versus static immersion (at $60^{\circ} \mathrm{C}$ ) $(\square)$ on the degree of PVC surface modification in $\mathrm{DMF} / \mathrm{H}_{2} \mathrm{O}$ systems with potassium carbonate as the base.

An increase in the degree of modification of both statically-immersed and ultrasonicallytreated films was observed as the solvent concentration was increased, as demonstrated in 
Fig. 3, due to enhanced penetration of the thiolate modifier into the expanded polymer chain network $[12,13]$. At concentrations of DMF higher than those presented here, the PVC dissolved, in accordance with previous reports [13].

The use of sonication at ambient temperature for modification of the PVC surface has been widely investigated, with metal ion chelation, and grafting of dyes such as Crystal Violet, Rose Bengal and Methylene Blue, and polymers such as PMMA, to PVC previously reported [16]. In addition to improving the mass transport of reagents, ultrasound was expected to enhance wetting of the PVC surface, thereby promoting contact between the PVC film and modifying agent, via high velocity impingement of the surface by microjets formed by the collapse of cavitation bubbles [16]. As displayed in Fig. 3, however, sonicated films exhibited lower degrees of surface modification compared to their statically-immersed counterparts, with these reductions becoming more pronounced as the water content of the reaction medium increased. For example, in $\mathrm{DMF} / \mathrm{H}_{2} \mathrm{O} 4: 1$ systems, the degree of modification of sonicated samples was four times lower than the corresponding staticallyimmersed samples. Furthermore, bombardment with base particulates caused pitting of the sonicated film surface, as displayed in Fig. 4. Due to the detrimental effect of sonication, even at ambient temperature, on integrity of film surfaces, and the inability to enhance the degree of chemical modification relative to that observed upon static immersion at $60^{\circ} \mathrm{C}$, samples were statically immersed in all further wet-chemical treatment studies.

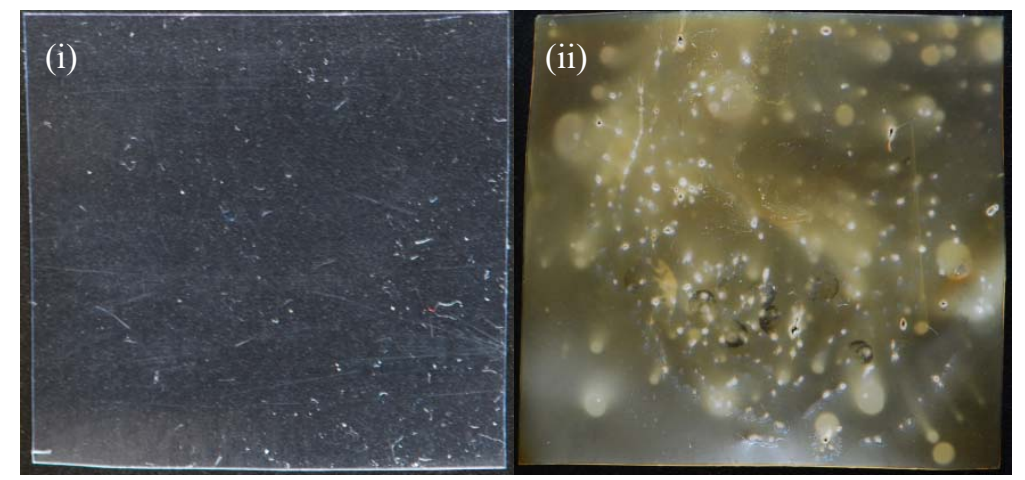

Fig. 4. Surfaces of (i) control PVC and (ii) 4-aminothiophenol-modified PVC following sonication in $\mathrm{DMF} / \mathrm{H}_{2} \mathrm{O}$ 6:1. The light spots represent surface irregularities caused by bombardment with base particulates.

\subsubsection{Effect of Base}

In the absence of a base, no variation in the IR spectra between treated and control PVC samples 
was observed, suggesting conversion of 4-aminothiophenol to the thiolate is a pre-requisite for nucleophilic substitution. The effect of two alternative deprotonating agents, potassium carbonate and caesium carbonate, with respective solubilities in DMF of $7.5 \mathrm{mgmL}^{-1}$ and $119.6 \mathrm{mgmL}^{-1}$ [17], on the degree of PVC surface modification can be seen in Fig. 5.

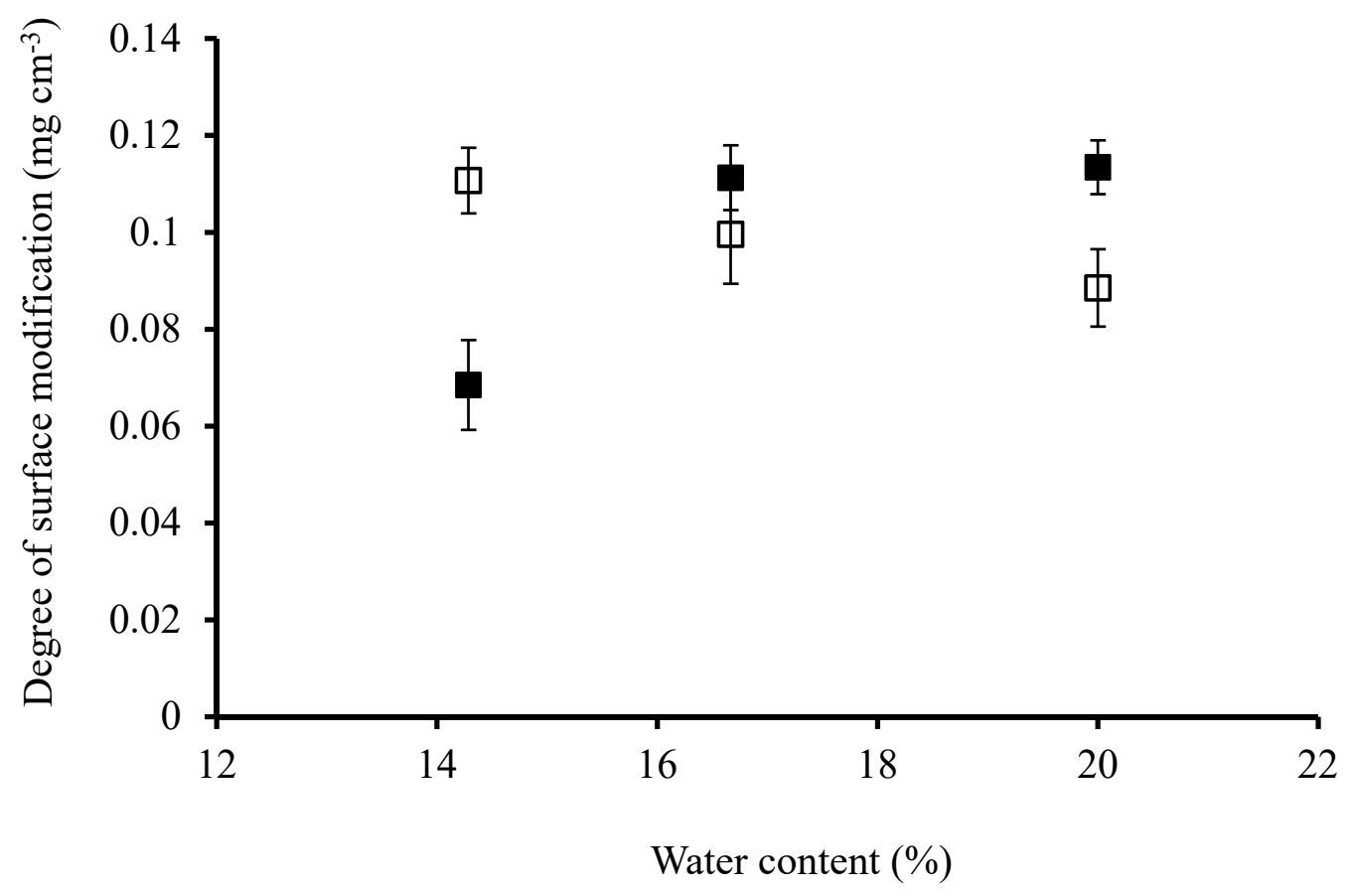

Fig. 5. The effect of potassium carbonate ( $\square$ ) and caesium carbonate ( $(\mathbf{)})$ on the degree of PVC surface modification in $\mathrm{DMF} / \mathrm{H}_{2} \mathrm{O}$ systems.

ATR-FTIR analysis of the modified films suggests that substitution of potassium carbonate by caesium carbonate does not have a substantial effect on the extent of PVC surface modification with 4-aminothiophenol under the conditions of this study, as graphically demonstrated in Fig. 5. The detrimental effect of potassium carbonate particulates on surface integrity has previously been shown by the images in Fig. 4, therefore the more soluble base, caesium carbonate, was employed in further studies, unless stated otherwise.

\subsubsection{Effect of Phase Transfer Catalyst}

Phase transfer catalysts (PTCs) function to facilitate transfer of the nucleophile from the aqueous phase to the organic phase, allowing intimate contact at the polymer-solvent interface, and thereby promoting substitution of chlorine atoms at the PVC surface [18]. The effect of tetrabutylammonium bromide (TBAB), an effective catalyst for dehydrochlorination of PVC 
[5], on the degree of PVC surface modification in $\mathrm{DMF} / \mathrm{H}_{2} \mathrm{O}$ systems with caesium carbonate as the base can be seen in Fig. 6.

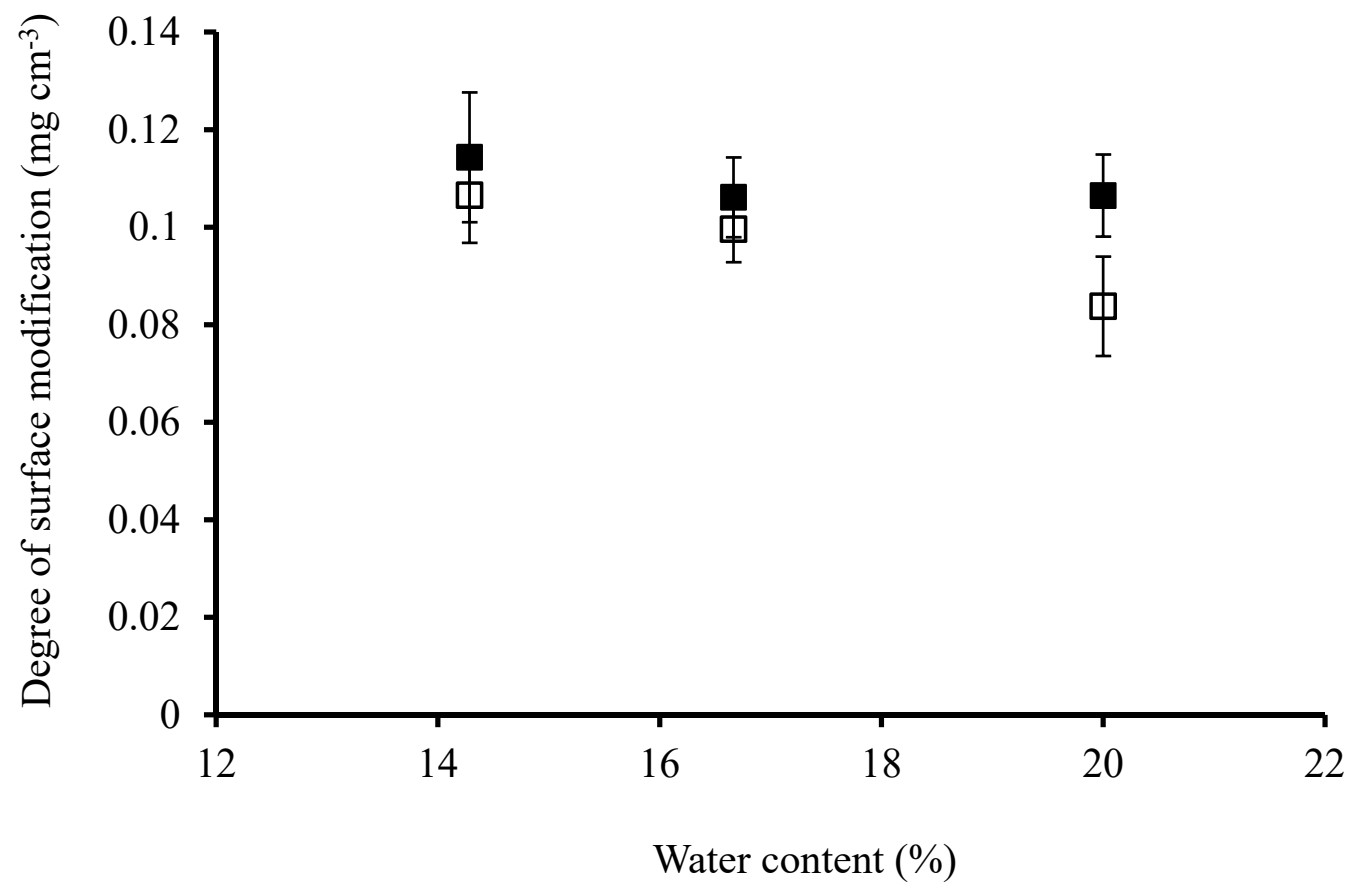

Fig. 6. Degree of PVC surface modification in $\mathrm{DMF} / \mathrm{H}_{2} \mathrm{O}$ systems with caesium carbonate in the absence ( $\square$ ) and presence ( $\mathbf{a})$ of TBAB.

Fig. 6 demonstrates that in the presence of TBAB as a PTC, the extent of PVC surface modification was up to $27 \%$ higher than in equivalent reaction media devoid of TBAB. The presence of a PTC in the basic $\mathrm{DMF} / \mathrm{H}_{2} \mathrm{O}$ systems was, however, detrimental to the mechanical integrity of the PVC samples, and induced surface irregularities can be seen in Fig. 7. Loss of optical transparency, with an associated increase in surface roughness, of PVC films has previously been reported during PTC-assisted surface modification by wet-chemical treatment [13].

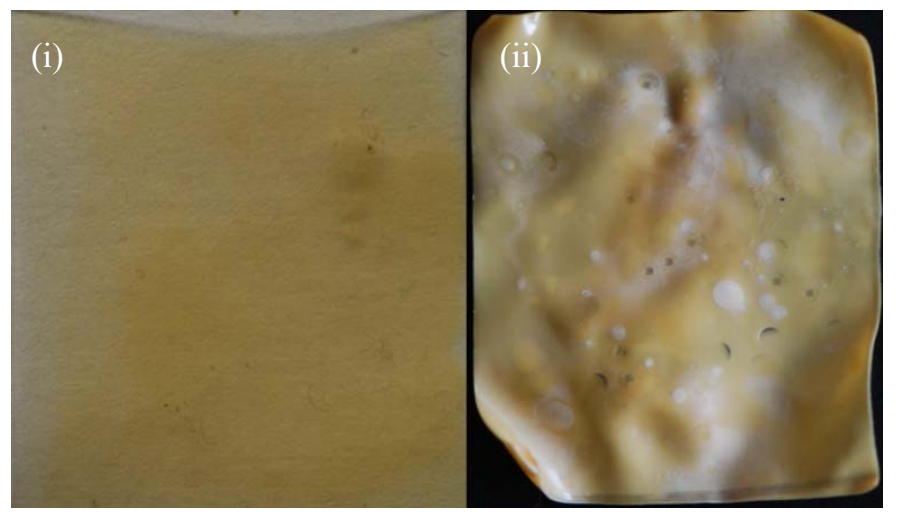


Fig. 7. PVC film modified in $\mathrm{DMF} / \mathrm{H}_{2} \mathrm{O} 6: 1$ in the absence (i) and presence (ii) of TBAB respectively.

This loss of mechanical integrity in the presence of TBAB was, however, found to be solvent-dependent, as reported below.

\subsubsection{Effect of Solvent}

A range of solvent systems have been successfully employed for chemical modification of powdered PVC via nucleophilic substitution, including tetrahydrofuran/dimethyl sulfoxide (THF/DMSO) [19] and $\mathrm{DMF} / \mathrm{H}_{2} \mathrm{O}$ [13], therefore the effect of alternative solvent compositions on the resultant degree of PVC surface modification was herein investigated.

Firstly, complete dissolution of the PVC film was observed within seconds of immersion in $\mathrm{THF} / \mathrm{H}_{2} \mathrm{O}$ systems, thus making this solvent unsuitable for surface modification of PVC. Secondly, no chemical modification of the PVC films occurred, as confirmed via spectroscopic characterisation, when ethylene glycol was employed as the solvent, irrespective of the base employed or presence of a PTC. In polar protic solvents, such as ethylene glycol, solvation of the nucleophile by hydrogen bonding occurs readily, thus hindering participation of this species in the nucleophilic substitution reaction. Thirdly, in systems employing DMSO/ $\mathrm{H}_{2} \mathrm{O}$ and potassium carbonate as the respective solvent and base, the extent of PVC surface modification was more than six-fold higher in the presence of TBAB than in equivalent solutions devoid of a PTC, as shown in Fig. 8. 


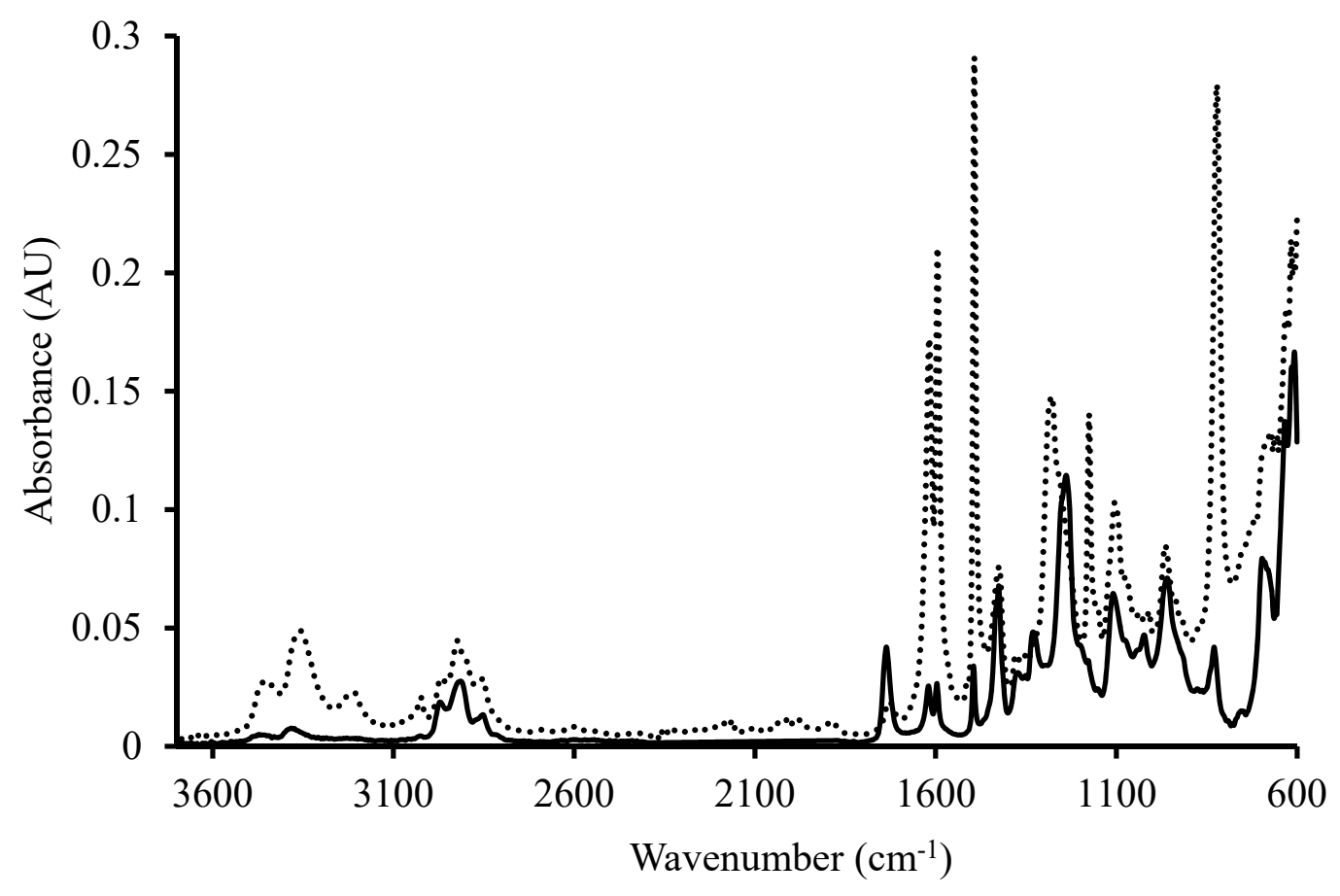

Fig. 8. Superimposed spectra of PVC films modified with 4-aminothiophenol in $\mathrm{DMSO} / \mathrm{H}_{2} \mathrm{O}$ 5:1 with potassium carbonate in the presence (...) and absence $(-)$ of TBAB.

In the absence of a PTC, PVC surfaces were modified to a lower extent in DMSO systems than in the corresponding DMF systems, as a result of reduced polymer chain swelling in the former solvent. Upon addition of TBAB (3.5 mol\%) to DMSO, however, approach of the nucleophile to the polymer surface was promoted, thus enhancing the resultant degree of surface modification. Furthermore, the modified films maintained their mechanical integrity in contrast to films modified in $\mathrm{DMF} / \mathrm{H}_{2} \mathrm{O}$ systems, which exhibited a warped and brittle nature due to extensive solvent-induced swelling of the polymer chains, as shown in the images in Fig. 7.

Increasing the quantity of TBAB from 3.5 to $7 \mathrm{~mol} \%$ resulted in a $25 \%$ increase in the degree of surface modification, as determined via spectroscopic characterisation. This increase was, however, accompanied by visual changes, including an intensified yellow colouration and cloudiness of the films, as observed in Fig. 9, attributed to partial crystallization of the polymer, as previously reported during chemical modification of PVC with sodium azide [13]. 


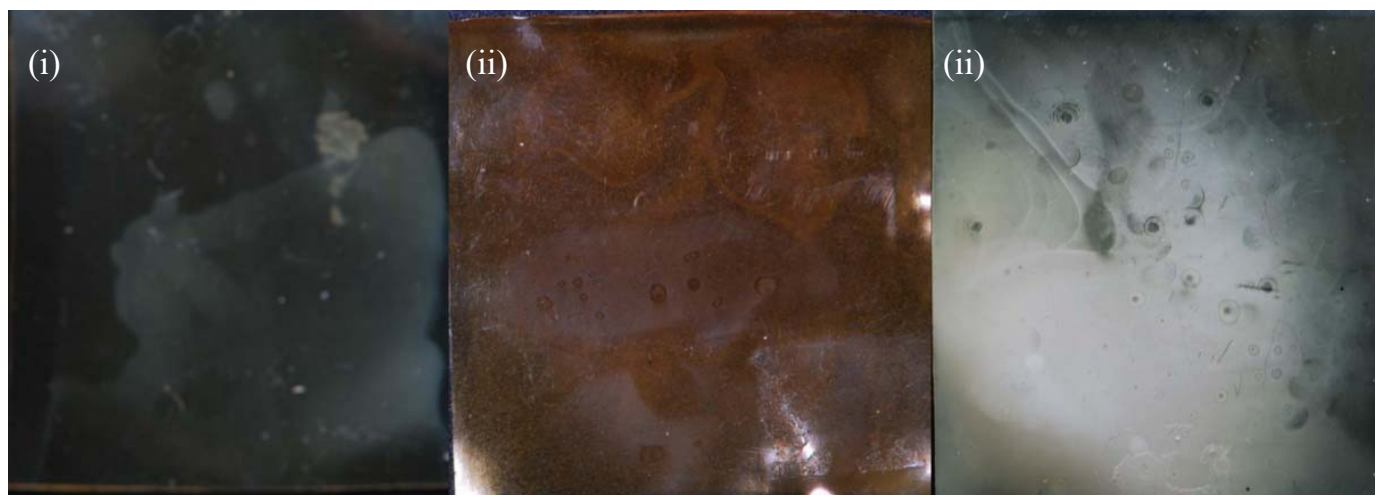

Fig. 9. PVC films modified with 4-aminothiophenol $(5 \mathrm{mmoL})$ at $60^{\circ} \mathrm{C}$ for $6 \mathrm{~h}$ in $\mathrm{DMSO} / \mathrm{H}_{2} \mathrm{O} 5: 1$ in the presence of caesium carbonate $(5 \mathrm{mmoL})$ and TBAB $(0,3.5$ and 7 mol\%) represented by (i), (ii) and (iii) respectively.

Furthermore, in DMSO solutions employing caesium carbonate as the base instead of potassium carbonate, degrees of surface modification were up to $12.5 \%$ higher, attributed to the greater solubility of the former base in DMSO (base solubilities were $362 \mathrm{mgmL}^{-1}$ and 47 $\mathrm{mgmL}^{-1}$ in DMSO respectively) [17]. These findings support a TBAB concentration of 3.5 mol\% and use of caesium carbonate as the base for optimal surface modification of PVC.

\subsection{Tensile Analysis}

Mechanical properties of the modified films, namely ultimate tensile strength, Young's modulus and percentage elongation at break, were determined following treatment in systems of $\mathrm{DMSO} / \mathrm{H}_{2} \mathrm{O}$ and $\mathrm{DMF} / \mathrm{H}_{2} \mathrm{O}$, with caesium carbonate and TBAB (3.5 mol\%), and results are reported in Table 1. No significant differences in ultimate tensile strength or Young's modulus of the PVC samples were demonstrated following treatment in DMSO/ $\mathrm{H}_{2} \mathrm{O}$ or $\mathrm{DMF} / \mathrm{H}_{2} \mathrm{O}$ systems, and optical transparency of the modified films was similar to unmodified PVC. In contrast, PVC modified in $\mathrm{DMF} / \mathrm{H}_{2} \mathrm{O}$ systems exhibited significantly lower elongation at break than control PVC and PVC treated in $\mathrm{DMSO} / \mathrm{H}_{2} \mathrm{O}$ systems. This reduced ductility was attributed to the higher levels of polymer chain entanglement in DMF, consequential to the extensive solvent-induced swelling. 
Table 1 Mechanical Properties of PVC Films Modified with 4-Aminothiophenol in the Presence of Caesium Carbonate and TBAB in $\mathrm{DMF} / \mathrm{H}_{2} \mathrm{O}$ and $\mathrm{DMSO} / \mathrm{H}_{2} \mathrm{O}$ Systems

\begin{tabular}{llll}
\hline PVC Reaction Media & $\begin{array}{l}\text { Ultimate Tensile } \\
\text { Strength }(\text { Mean } \pm \mathrm{SD})\end{array}$ & $\begin{array}{l}\text { Young's Modulus } \\
(\mathrm{Mean} \pm \mathrm{SD})\end{array}$ & $\begin{array}{l}\text { Elongation at Break } \\
(\mathrm{Nean} \pm \mathrm{SD})\end{array}$ \\
& $\left(\mathrm{N} \mathrm{mm}^{-2}\right)$ & $63.7 \pm 2.1$ & $(\%)$ \\
\hline Unmodified (control) & $34.8 \pm 1.3$ & $70.8 \pm 15.4$ & $149.2 \pm 8.8$ \\
$\mathrm{DMF} / \mathrm{H}_{2} \mathrm{O}$ & $36.6 \pm 4.4$ & $64.1 \pm 9.3$ & $23.2 \pm 4.8$ \\
$\mathrm{DMSO} / \mathrm{H}_{2} \mathrm{O}$ & $32.1 \pm 1.8$ & $127.3 \pm 11.0$ \\
\hline
\end{tabular}

\subsection{Kinetic Study of PVC Surface Modification with 4-Aminothiophenol}

Through systematic investigation of varying reaction conditions, we have demonstrated that efficient thiol attachment to $\mathrm{PVC}$, without compromising mechanical integrity, occurs in the presence of TBAB (3.5 mol\%) and caesium carbonate in a solvent system of DMSO/ $\mathrm{H}_{2} \mathrm{O}$ 5:1. Kinetics of the PVC surface modification reaction were then investigated in the optimised reaction conditions by statically immersing PVC samples in media containing the 4aminothiophenol modifying agent for time periods ranging from 2 to $42 \mathrm{~h}$. Resultant degrees of surface modification were determined by FTIR, as previously described, using absorption bands of 4-aminothiophenol aromatic vibrational modes. The maximum degree of modification was quantified after $12 \mathrm{~h}$ as $149.7 \mu \mathrm{g} \mathrm{cm}^{-3}$. 


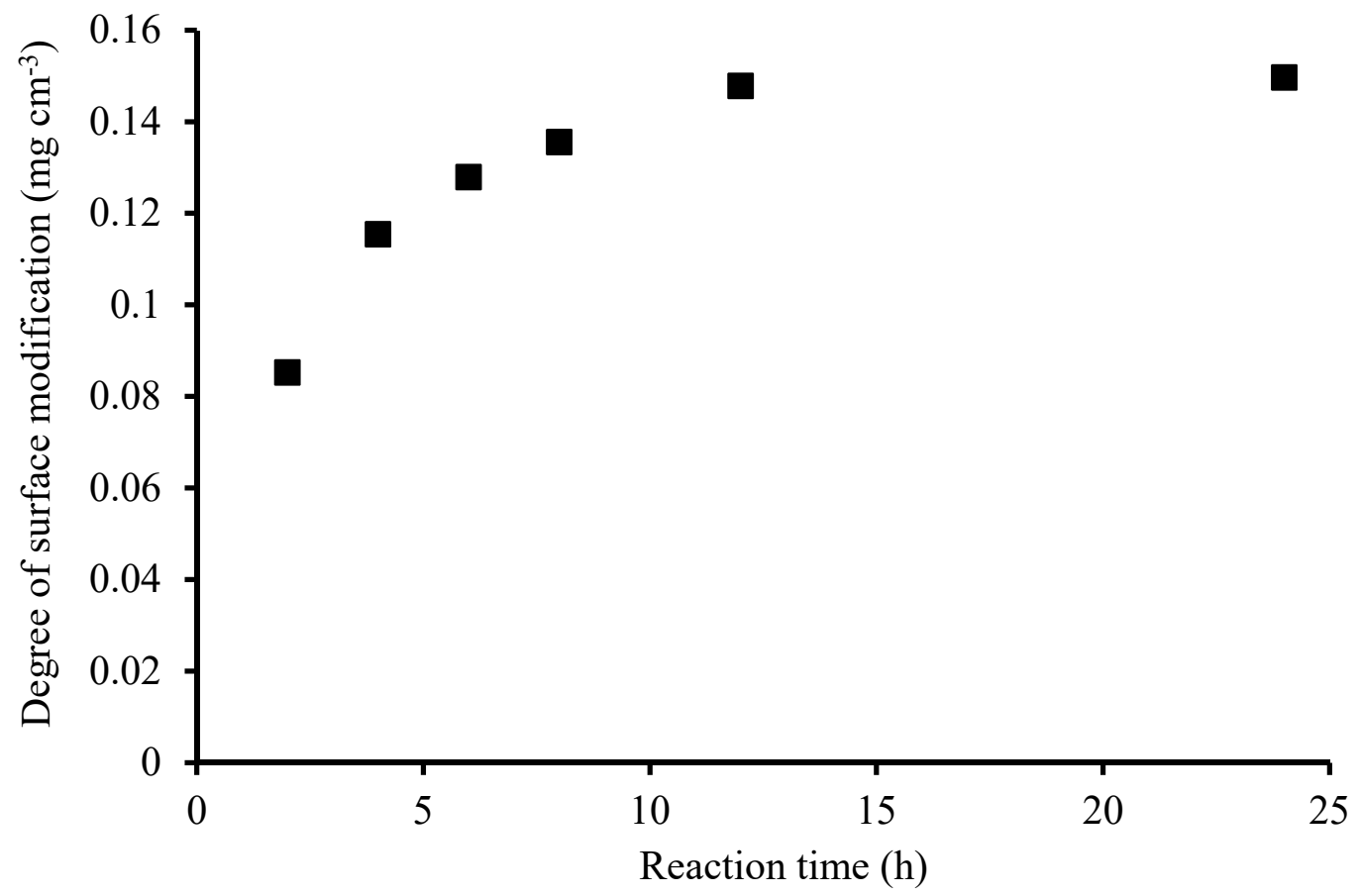

Fig. 10. Kinetics of the PVC surface modification reaction with 4-aminothiophenol in DMSO/ $\mathrm{H}_{2} \mathrm{O} 5: 1$ with caesium carbonate and TBAB (3.5 mol\%).

As demonstrated in Fig. 10, at time periods exceeding $12 \mathrm{~h}$, the degree of surface modification appeared to plateau as a result of the surface selectivity of FTIR spectroscopy in the ATR mode. Bulk modification was, however, visually evident upon prolonged reaction conditions by intensified colouration of the PVC films, corresponding to the formation of conjugated $\mathrm{C}=\mathrm{C}$ double bonds during the dechlorination of PVC, as previously reported $[5,20,21]$. PVC should therefore be statically immersed in the reaction media for durations of less than $12 \mathrm{~h}$ to prevent loss of polymer mechanical integrity.

\section{Conclusions}

We herein present an optimised method for efficient surface functionalization of PVC with thiol moieties, determined through systematic investigation of the effect of variable reaction conditions, including solvent composition, sonication, and the presence of base and/or PTC, on mechanical integrity and degree of surface modification of PVC films. Sonication introduced surface irregularities, therefore samples should be statically immersed for reaction times of less than $12 \mathrm{~h}$ to maintain surface and bulk mechanical integrity of the polymer films. Detrimental effects on surface integrity were, in addition, demonstrated upon employment of potassium carbonate as the base, whereas control of the solvent composition 
was important for maintenance of bulk mechanical integrity. Alternative solvent systems comprising $\mathrm{THF} / \mathrm{H}_{2} \mathrm{O}$ and $\mathrm{DMF} / \mathrm{H}_{2} \mathrm{O}$ resulted in dissolution and reduced ductility of the films respectively. Through this study, we can, importantly, conclude that optimal levels of surface modification approximating $150 \mu \mathrm{g} \mathrm{cm}^{-3}$, with no loss of surface smoothness, were observed upon static immersion of PVC in the presence of the PTC, TBAB, with employment of caesium carbonate and DMSO as the base and solvent, respectively. This efficient method for thiol-functionalization of PVC will ultimately allow the performance of this widely used biomaterial to be enhanced through facilitation of a range of property-enhancing surface modifications.

\section{Acknowledgements}

Funding: This work was supported by the Engineering and Physical Sciences Research

Council (EPRSC), U.K., [grant number EP/H012249/1], and the use of the EPSRC-sponsored Durham Solid State NMR Research Service (Durham, U.K.) is gratefully acknowledged.

\section{References}

1. S. Moulay, Prog. Polym. Sci., 2010, 35, 303-331.

2. K. Mulder, M. Knot, Technol. Soc., 2001, 23, 265-286.

3. L. Bernard, R. Cueff, D. Bourdeaux, C. Breysse, V. Sautou, Anal. Bioanal. Chem., 2015, 407, 1651-1659.

4. S. Lakshmi, A. Jayakrishnan, Polymer, 1998, 39, 151-157.

5. T. Kameda, G. Grause, T. Yoshioka, Mater. Chem. Phys., 2010, 124, 163-167.

6. C. Liu, Y. Luo, B. Zhong, S. Li, B. Guo, D. Jia, Express Polym. Lett., 2011, 5, 591-603.

7. Y. Zou, J. Kizhakkedathu, D. Brooks, Macromolecules, 2009, 42, 3258-3268.

8. J. Milenkovic, J. Hrenovic, I. Goic-Barisic, M. Tomic, J. Djonlagic, N. Rajic, Biofouling, 
2014, 30, 965-973.

9. M. Ghoranneviss, S. Shahidi, J. Wiener, Plasma Sci. Technol., 2010, 12, 204-207.

10. Y. Haishima, K. Isama, C. Hasegawa, T. Yuba, A. Matsuoka, J. Biomed. Mater. Res. A, 2013, 101, 2630-2643.

11. T. Kameda, M. Ono, G. Grause, T. Mizoguchi, T. Yoshioka, Polym. Degrad. Stabil., 2009, 94, 107-112.

12. J. Reyes-Labarta, M. Herrero, P. Tiemblo, C. Mijangos, H. Reinecke, Polymer, 2003, 44, 2263-2269.

13. J. Sacristán, H. Reinecke, C. Mijangos, Polymer, 2000, 41, 5577-5582.

14. C. McCoy, J. Cowley, S. Gorman, G. Andrews, D. Jones, J. Pharm. Pharmacol., 2009, 61, 1163-1169.

15. J. Coates, in Encyclopaedia of Analytical Chemistry, ed. R. Meyers, Wiley, Chichester, $1^{\text {st }}$ ed., 2000, Interpretation of Infrared Spectra, A Practical Approach, 10815-10837.

16. G. Price, A. Clifton, Polym. Int., 1999, 48, 1141-1146.

17. R. Kellog, Science of Synthesis: Houben-Weyl Methods of Molecular Transformations, Organometallics: Compounds of Group 1, Georg Thieme Verlag, Stuttgart, 2014.

18. S. Marian, G. Levin, J. Appl. Polym. Sci., 1981, 26, 3295-3304.

19. N. Bicak, D. Sherrington, H. Bulbul, Eur. Polym. J., 2001, 37, 801-805

20. T. Kameda, M. Ono, G. Grause, T. Mizoguchi, T. Yoshioka, Polym. Eng. Sci., 2010, 50, 6975.

21. Lewis G.N., Calvin M., Chem. Rev. 1939, 25, 273-328. 